\title{
GOOD APPROXIMATIONS AND CONTINUED FRACTIONS
}

\author{
COR KRAAIKAMP AND PIERRE LIARDET
}

(Communicated by William Adams)

\begin{abstract}
Let $\left(q_{n}\right)_{n}$ be the sequence of best approximation denominators of an irrational number $\alpha$. The set of real numbers $x$ for which $q_{n} x \rightarrow 0$ $(\bmod 1)$ is studied. It is shown that a number $x$ belongs to $\alpha \mathbb{Z}(\bmod 1)$ if and only if a simple condition on the speed of the convergence related to an arithmetic property of $\alpha$ is satisfied. This set is uncountable whenever $\alpha$ has unbounded partial quotients.
\end{abstract}

\section{INTRODUCTION}

Let $\alpha$ be a real irrational number with regular continued fraction expansion $\left[a_{0} ; a_{1}, \ldots, a_{n}, \ldots\right]$. Let $\left(q_{n}(\alpha)\right)_{n \geq-1}$ be the sequence of denominators of the regular convergents $p_{n}(\alpha) / q_{n}(\alpha)$ of $\alpha$, given by

$$
q_{-1}(\alpha):=0, \quad q_{0}(\alpha):=1, \quad q_{n}(\alpha):=a_{n} q_{n-1}(\alpha)+q_{n-2}(\alpha), \quad n \geq 1 .
$$

In the sequel we will write, for short, $q_{n}$ and $p_{n} / q_{n}$ instead of $q_{n}(\alpha)$ and $p_{n}(\alpha) / q_{n}(\alpha)$, respectively.

We are interested in the set $E(\alpha)$ of real numbers $\beta$ for which the sequence $\left(q_{n} \beta\right)_{n \geq 1}$ converges, modulo 1 , to 0 . Clearly $E(\alpha)$ is an additive subgroup of $\mathbb{R}$ which contains $\alpha \mathbb{Z}+\mathbb{Z}$. From a result of Eggleston [E], if $\alpha$ has bounded partial quotients, then $E(\alpha)$ is at most enumerable. In this particular case a result of Larcher [L] states that $E(\alpha)=\alpha \mathbb{Z}+\mathbb{Z}$. We will generalize this result. Let $\|\xi\|$ be the distance of $\xi$ from the nearest integer and put $\theta_{n}=\theta_{n}(\alpha):=q_{n}\left|q_{n} \alpha-p_{n}\right|$, $n \geq 0$. Let $\beta$ be a real number. We will show then, among other things, that for any irrational number $\alpha$ we have $\beta \in \alpha \mathbb{Z}+\mathbb{Z}$ if and only if $\left\|q_{n} \beta\right\| \in o\left(\theta_{n}\right)$, $(n \rightarrow \infty)$.

\section{SOME NOTATIONS}

Put

$$
A_{0}:=\left[\begin{array}{cc}
1 & a_{0} \\
0 & 1
\end{array}\right], \quad A_{n}:=\left[\begin{array}{cc}
0 & 1 \\
1 & a_{n}
\end{array}\right], \quad n \geq 1,
$$

Received by the editors October 1, 1989 and, in revised form, June 29, 1990.

1980 Mathematics Subject Classification (1985 Revision). Primary 11J70, 11 A55.

Key words and phrases. Continued fractions, diophantine approximations. 
and

$$
M_{n}:=A_{0} \cdots A_{n}, \quad n \geq 0 .
$$

A short calculation then yields

$$
M_{n}=\left[\begin{array}{ll}
p_{n-1} & p_{n} \\
q_{n-1} & q_{n}
\end{array}\right], \quad n \geq 0 .
$$

We consider $M_{n}$ as the linear transformation $x \rightarrow\left(p_{n-1} x+p_{n}\right) /\left(q_{n-1} x+q_{n}\right)$, so that

We furthermore define

$$
\frac{p_{n}}{q_{n}}=M_{n}(0), \quad n \geq 0
$$

$$
T_{n}=\left[0 ; a_{n+1}, a_{n+2}, \ldots\right], \quad n \geq 0,
$$

and

We have

$$
V_{n}:=\frac{q_{n-1}}{q_{n}}, \quad n \geq 0
$$

$$
\begin{gathered}
V_{n}=\left[0 ; a_{n}, \ldots, a_{1}\right], \quad n \geq 1, \quad V_{0}=0, \\
\alpha=\frac{p_{n}+T_{n} p_{n-1}}{q_{n}+T_{n} q_{n-1}}, \quad n \geq 0,
\end{gathered}
$$

and

$$
\theta_{n}=\frac{T_{n}}{1+T_{n} V_{n}}, \quad n \geq 0
$$

Notice that $\theta_{n}=q_{n}\left\|q_{n} \alpha\right\|$. Other useful properties are

(4) $\quad\left|q_{n} \alpha-p_{n}\right|<\frac{1}{q_{n+1}}$ and $q_{n+1}\left\|q_{n} \alpha\right\|+q_{n}\left\|q_{n+1} \alpha\right\|=1, \quad n \geq 0$;

see $[P, K]$.

Lemma. Let $A, B \in \mathbb{Z}$ and define the real number $\beta$ by

$$
\beta:=\frac{A T_{n}+B}{q_{n-1} T_{n}+q_{n}},
$$

where $n \geq 0$. Then $\beta=\left(\delta_{n} \Delta_{n}\right) \alpha-\delta_{n}\left(A p_{n}-B p_{n-1}\right)$, where

$$
\delta_{n}:=\operatorname{det} M_{n}=(-1)^{n}, \quad n \geq 0, \quad \Delta_{n}:=\operatorname{det}\left[\begin{array}{cc}
A & B \\
q_{n-1} & q_{n}
\end{array}\right] \text {. }
$$

Proof. From (2) and the definition of $\beta$ we have $\beta=M_{n}^{*} M_{n}^{-1}(\alpha)$, where

But now the result follows at once, since

$$
M_{n}^{*}:=\left[\begin{array}{cc}
A & B \\
q_{n-1} & q_{n}
\end{array}\right] .
$$

$$
\begin{aligned}
M_{n}^{*} M_{n}^{-1} & =\delta_{n}\left[\begin{array}{cc}
A & B \\
q_{n-1} & q_{n}
\end{array}\right]\left[\begin{array}{cc}
q_{n} & -p_{n} \\
-q_{n-1} & p_{n-1}
\end{array}\right] \\
& =\delta_{n}\left[\begin{array}{cc}
\Delta_{n} & -A p_{n}+B p_{n-1} \\
0 & \delta_{n}
\end{array}\right] \cdot \square
\end{aligned}
$$


Theorem 1. Let $\alpha$ be an irrational number. For each real number $\beta$ we have

$$
\beta \in \alpha \mathbb{Z}+\mathbb{Z} \Leftrightarrow \exists n_{0}=n_{0}(\beta), \quad \forall n \geq n_{0}:\left\|q_{n} \beta\right\| \leq \frac{1}{4} \theta_{n} .
$$

Proof. Let $\beta=a \alpha+b$, for some $a, b \in \mathbb{Z}$. Then

$$
\left\|q_{n} \beta\right\| \leq|a|\left\|q_{n} \alpha\right\|=\frac{|a|}{q_{n}} q_{n}\left\|q_{n} \alpha\right\|=\frac{|a|}{q_{n}} \theta_{n}
$$

and the implication follows directly from the fact that the sequence of denominators $\left(q_{n}\right)_{n \geq 0}$ is a monotonically increasing sequence of positive integers.

Now let $n \in \mathbb{N}$. For $k=0,1, \ldots, q_{n}-1$ we define the closed intervals $I_{n}^{k}=I_{n}^{k}(\alpha)$ by

$$
I_{n}^{k}:=\left[\frac{k}{q_{n}}-\frac{\theta_{n}}{4 q_{n}}, \frac{k}{q_{n}}+\frac{\theta_{n}}{4 q_{n}}\right],
$$

with $\theta_{n}=\theta_{n}(\alpha), q_{n}=q_{n}(\alpha)$. We call $n$ the rank of $I_{n}^{k}$. Since $0<\theta_{n}<1$ for all $n$, it is obvious that two different intervals of the same rank are disjoint. Now suppose that an interval of rank $n$ intersects with two intervals of rank $n+1$. This would give

$$
\frac{2 \theta_{n+1}}{4 q_{n+1}}+\frac{2 \theta_{n}}{4 q_{n}} \geq \frac{1}{q_{n+1}}
$$

i.e.,

$$
\theta_{n+1}+\frac{q_{n+1}}{q_{n}} \theta_{n} \geq 2 \text {. }
$$

A direct consequence of (4) is that $\left(\theta_{n} q_{n+1}\right) / q_{n}<1$ for all $n$, hence we are led to a contradiction. The conclusion is that an interval of rank $n$ intersects with at most one interval of rank $n+1$. Now let $\beta$ be a real number with the property that there exists a positive integer $n_{0}=n_{0}(\beta)$ such that

$$
\left\|q_{n} \beta\right\| \leq \frac{1}{4} \theta_{n}, \quad \text { for all } n \geq n_{0} .
$$

Without any loss of generality we may assume that $0 \leq \beta<1$. Then for every $n \geq n_{0}$ there exists a unique $k(n)=k(n, \beta), k(n) \in\left\{0, \ldots, q_{n}-1\right\}$, such that

$$
\beta \in I_{n}^{k(n)} \text {. }
$$

Next we introduce the number $\beta^{*}$, defined by

$$
\beta^{*}:=\frac{k\left(n_{0}\right) T_{n_{0}+1}+k\left(n_{0}+1\right)}{q_{n_{0}} T_{n_{0}+1}+q_{n_{0}+1}} .
$$

From the lemma

$$
\beta^{*}=\delta_{n_{0}+1} \Delta_{n_{0}+1} \alpha-\delta_{n_{0}+1}\left(k\left(n_{0}\right) p_{n_{0}+1}-k\left(n_{0}+1\right) p_{n_{0}}\right) ;
$$

in particular we have $\beta^{*} \in \alpha \mathbb{Z}+\mathbb{Z}$. For abbreviation we will write $\Delta$ instead of $\Delta_{n_{0}+1}$. Notice that (5) yields

$$
\left\|q_{n} \beta^{*}\right\| \leq \frac{|\Delta|}{q_{n}} \theta_{n} .
$$


Next observe that

$$
\begin{aligned}
\left|\frac{k\left(n_{0}\right)}{q_{n_{0}}}-\frac{k\left(n_{0}+1\right)}{q_{n_{0}+1}}\right| & \leq\left|\beta-\frac{k\left(n_{0}\right)}{q_{n_{0}}}\right|+\left|\beta-\frac{k\left(n_{0}+1\right)}{q_{n_{0}+1}}\right| \\
& \leq \frac{1}{4}\left(\frac{\theta_{n_{0}}}{q_{n_{0}}}+\frac{\theta_{n_{0}+1}}{q_{n_{0}+1}}\right) .
\end{aligned}
$$

From this it follows that

$$
|\Delta| \leq \frac{1}{4} q_{n_{0}} q_{n_{0}+1}\left(\frac{\theta_{n_{0}}}{q_{n_{0}}}+\frac{\theta_{n_{0}+1}}{q_{n_{0}+1}}\right) .
$$

Hence

$$
\frac{|\Delta|}{q_{n_{0}+1}} \leq \frac{1}{4}\left(\theta_{n_{0}}+\frac{q_{n_{0}}}{q_{n_{0}+1}} \theta_{n_{0}+1}\right)=\frac{1}{4}\left(q_{n_{0}}\left\|q_{n_{0}} \alpha\right\|+q_{n_{0}}\left\|q_{n_{0}+1} \alpha\right\|\right),
$$

therefore

$$
|\Delta| \leq \frac{1}{4} q_{n_{0}+1} .
$$

This combined with (7) shows that $\left\|q_{n} \beta^{*}\right\| \leq \frac{1}{4} \theta_{n}$, for all $n>n_{0}$. In the same way as we showed (6) we find that there exists a sequence of intervals $I_{n}^{k^{*}(n)}$, such that $\beta^{*} \in I_{n}^{k^{*}(n)}$ for all $n>n_{0}$.

The linear transformation

$$
f: x \rightarrow \frac{k\left(n_{0}\right) x+k\left(n_{0}+1\right)}{q_{n_{0}} x+q_{n_{0}+1}}
$$

is strictly monotonic on $[0,1]$, hence

$$
|\beta-f(0)| \leq|f(1)-f(0)|=\frac{|\Delta|}{q_{n_{0}+1}\left(q_{n_{0}}+q_{n_{0}+1}\right)}<\frac{1}{4} \frac{1}{q_{n_{0}+1}},
$$

the last inequality in view of (8). This means that $I_{n}^{k(n)}=I_{n}^{k^{*}(n)}$ for all $n>n_{0}$, i.e. $\beta, \beta^{*} \in I_{n}^{k(n)}, n \geq n_{0}$, so that $\beta^{*}=\beta$. Hence $\beta \in \alpha \mathbb{Z}+\mathbb{Z}$.

Remark 1. It follows from (5) that $\beta \in \alpha \mathbb{Z}+\mathbb{Z}$ implies $\left\|q_{n} \beta\right\| \in o\left(\theta_{n}\right)$. Reciprocally, if $\left\|q_{n} \beta\right\| \in O\left(\theta_{n}\right)$, then there exists a positive integer $n_{0}$ such that $\left\|q_{n} \beta\right\| \leq \frac{1}{4} \theta_{n}$, for all $n \geq n_{0}$. From Theorem 1 we get $\beta \in \alpha \mathbb{Z}+\mathbb{Z}$. An equivalent formulation for Theorem 1 is therefore

Theorem $1^{\prime}$. Let $\alpha$ be an irrational number. For each real number $\beta$ we have

$$
\beta \in \alpha \mathbb{Z}+\mathbb{Z} \Leftrightarrow\left\|q_{n} \beta\right\| \in o\left(\theta_{n}\right), \quad(n \rightarrow \infty) .
$$

Remark 2. Assume now that $\alpha$ is an irrational number with bounded partial quotients, i.e., there exists a positive integer $K$ such that $a_{n} \leq K$, for $n \geq 1$. From formula (3) we derive $\theta_{n} \geq 1 /(K+2)$. This implies the following result: 
Corollary [L]. Let $\alpha$ be an irrational number with bounded partial quotients. Then

$$
\beta \in \alpha \mathbb{Z}+\mathbb{Z} \Leftrightarrow \lim _{n \rightarrow \infty}\left\|q_{n} \beta\right\|=0 .
$$

In fact, we can replace the limit condition by the following weaker form:

$$
\limsup _{n \rightarrow \infty}\left\|q_{n} \beta\right\|<\frac{1}{4} \frac{1}{K+2} .
$$

Remark 3. A theorem of Stewart [S, Theorem 3] yields that, in case $\alpha$ has bounded partial quotients, the relation $\beta \in \alpha \mathbb{Z}+\mathbb{Z}$ is equivalent to

$$
\limsup _{n \rightarrow \infty}\left(2\left\|q_{n} \beta\right\|-\theta_{n}\right) \leq 0 .
$$

This last form is stronger that the previous one, however the proof given by Stewart is technically quite involved. Moreover, from the construction given in Theorem 3 we will see that in case $\alpha$ has not bounded partial quotients, the inequality $(*)$ is not equivalent to $\beta \in \alpha \mathbb{Z}+\mathbb{Z}$. Now we improve Larcher's result.

Theorem 2. Let $\alpha$ have bounded partial quotients. Then a real number $\beta$ belongs to $\alpha \mathbb{Q}+\mathbb{Q}$ if and only if the sequence $\left(\left\|q_{n} \beta\right\|\right)_{n}$ has only finitely many limit points.

Proof. Clearly if $\beta=(p \alpha+r) / q(p, q, r \in \mathbb{Z}, q \neq 0)$ then the sequence $\left(\left\|q_{n} \beta\right\|\right)_{n}$ has limit points in $\{0,1 / q, \ldots,(q-1) / q\}$. Conversely, let $S$ be the set of limit points of the above sequence. By the Kronecker approximation theorem there exists a positive integer $m$ such that

$$
\forall s \in S:\|m \cdot s\|<\frac{1}{8(K+2)} .
$$

By assumption, for any $\varepsilon>0$ there exists an integer $N(\varepsilon)$ satisfying

$$
(\forall n \in \mathbb{N})\left(n \geq N(\varepsilon) \Rightarrow \exists s_{n} \in S:\left\|q_{n} \cdot \beta-s_{n}\right\| \leq \varepsilon\right) .
$$

On the other hand, we have

$$
\begin{aligned}
\|m n \cdot \beta\| & \leq \min _{r, t \in \mathbb{N}}\left(\left|m\left(q_{n} \cdot \beta-r-s_{n}\right)\right|+\left|m \cdot s_{n}-t\right|\right) \\
& \leq m\left\|q_{n} \cdot \beta-s_{n}\right\|+\left\|m \cdot s_{n}\right\| .
\end{aligned}
$$

Choose $\varepsilon=1 / 16 m(K+2)$. Then, for sufficiently large $n$,

and Remark 2 finishes the proof.

$$
\left\|m q_{n} \cdot \beta\right\|<\frac{1}{4} \frac{1}{K+2}
$$

In [L] it is shown that there exist real numbers $\alpha$ and $\beta, \beta \notin \alpha \mathbb{Z}+\mathbb{Z}$, such that $\lim _{n \rightarrow \infty}\left\|q_{n} \beta\right\|=0$. Notice that, in view of the above corollary, the number $\alpha$ has unbounded partial quotients. Here we will show that for each irrational number $\alpha$ with unbounded partial quotients there exist uncountably many real numbers $\beta$ such that $\lim _{n \rightarrow \infty}\left\|q_{n} \beta\right\|=0$. In fact if $\lim _{n \rightarrow \infty} q_{n+1} / q_{n}=+\infty$ then the Hausdorff dimension of $E(\alpha)$ is equal to 1 (see [E]). But for almost all $\alpha$ one has $\liminf \operatorname{in}_{n \rightarrow \infty} q_{n+1} / q_{n}=1$ and $\lim \sup _{n \rightarrow \infty} q_{n+1} / q_{n}=+\infty$. 
Theorem 3. Let $\alpha$ be an irrational number with unbounded partial quotients. Then the additive subgroup $E(\alpha)$ of $\mathbb{R}$ defined by

is uncountable.

$$
E(\alpha):=\left\{\beta \in \mathbb{R} ; \lim _{n \rightarrow \infty}\left\|q_{n} \beta\right\|=0\right\},
$$

Proof. Let $\left[a_{0} ; a_{1}, \ldots, a_{n}, \ldots\right]$ be the regular continued fraction expansion of $\alpha$. Then there exists an infinite subsequence $\left(n_{l}\right)_{l \geq 1}$ of positive integers such that for all $l \geq 1, a_{n_{l}+1}>2^{l}$. Due to the well-known fact that

$$
T_{n} \in\left(1 /\left(a_{n+1}+1\right), 1 / a_{n+1}\right) \text { for all } n \geq 0,
$$

we have, for all $l \geq 1, T_{n_{l}}<1 / a_{n_{l}+1}<2^{-l}$. From (1), respectively (3), we moreover have, for all $l \geq 1, V_{n_{l}+1}<1 / a_{n_{l}+1}<2^{-l}$ and $\theta_{n_{l}}<T_{n_{l}}<2^{-l}$. Define the real number $\beta$ by

$$
\beta:=\sum_{l=1}^{\infty} \varepsilon_{l}\left(q_{n_{l}} \alpha-P_{n_{l}}\right), \quad \text { where } \varepsilon_{l} \in\{0,1\}, l \geq 1 .
$$

Notice that $\beta$ is well defined and the series absolutely converges. Moreover, different sequences $\left(\varepsilon_{l}\right)_{l}$ lead to different $\beta$. In fact, from usual recurrent relations satisfying by sequences $\left(q_{n}\right)_{n}$ and $\left(p_{n}\right)_{n}$, we get $\sum_{k=1}^{\infty} a_{m+2 k+1}\left\|q_{m+2 k} \alpha\right\|=$ $\left\|q_{m+1} \alpha\right\|$. Hence for $\eta_{l} \in\{0,1,-1\}, l \geq k$ and $\eta_{k}=1$, one has

$$
\sum_{l=k}^{\infty} \eta_{l}\left(q_{n_{l}} \alpha-p_{n_{l}}\right) \geq\left\|q_{n_{k}} \alpha\right\|-\sum_{l=k+1}^{\infty}\left\|q_{n_{l}} \alpha\right\|>0 .
$$

We claim that $\beta \in E(\alpha)$, from which the theorem follows.

Let $m$ and $n$ be two positive integers. Put $\Delta(m, n):=\left(p_{m} q_{n}-p_{n} q_{m}\right)$. Then

$$
q_{n}\left(q_{m} \alpha-p_{m}\right)+\Delta(n, m)=q_{m}\left(q_{n} \alpha-p_{n}\right) .
$$

Now let $n$ be a positive integer, $n>n_{3}$. Then there exists a unique positive integer $k=k(n) \geq 3$ such that $n_{k}<n \leq n_{k+1}$. In order to show that $\beta \in E(\alpha)$ it is now sufficient to show that we have $\left\|q_{n} \beta\right\|<3 / 2^{k}$, since $k$ and $n$ go to infinity simultaneously.

Suppose that $l \leq k$. Then $n_{l}<n$. Put $C_{l}:=\Delta\left(n, n_{l}\right)$. Applying the above with $m=n_{l}$ and putting

$$
\beta(k):=\sum_{l \leq k} \varepsilon_{l}\left(q_{n_{l}} \alpha-p_{n_{l}}\right), \quad S_{k}:=\sum_{l \leq k} \varepsilon_{l} C_{l}
$$

we get

$$
\left|q_{n} \beta(k)-S_{k}\right| \leq \sum_{l \leq k} \varepsilon_{l} q_{n_{l}}\left\|q_{n} \alpha\right\| \leq \sum_{l \leq k} \varepsilon_{l} \frac{q_{n_{l}}}{q_{n}} .
$$

Since $n_{l} \leq n_{k}<n$ and

$$
\begin{aligned}
\frac{q_{n_{l}}}{q_{n}} & =\frac{q_{n_{l}}}{q_{n_{l}+1}} \frac{q_{n_{l}+1}}{q_{n_{l}+2}} \cdots \frac{q_{n-1}}{q_{n}} \\
& \leq V_{n_{l}+1} V_{n_{l+1}+1} \cdots V_{n_{k}+1}<2^{-l} 2^{-l-1} \cdots 2^{-k}
\end{aligned}
$$


we find that

$$
0 \leq \sum_{l \leq k} \varepsilon_{l} \frac{q_{n_{l}}}{q_{n}} \leq \sum_{l \leq k} 2^{-l} 2^{-l-1} \cdots 2^{-k}<\frac{1}{2^{k-1}}
$$

Hence

$$
\left|q_{n} \beta(k)-S_{k}\right|<2^{1-k}
$$

Now we estimate the sum $q_{n} \sum_{l \geq k+1} \varepsilon_{l}\left\|q_{n_{l}} \alpha\right\|$. For $l \geq k+1$ one has $q_{n} \leq q_{n_{l}}$, and therefore

$$
0 \leq q_{n} \sum_{l \geq k+1} \varepsilon_{l}\left\|q_{n_{l}} \alpha\right\| \leq \sum_{l \geq k+1} q_{n_{l}}\left\|q_{n_{l}} \alpha\right\|=\sum_{l \geq k+1} \theta_{n_{l}}<\sum_{l \geq k+1} \frac{1}{2^{l}}=\frac{1}{2^{k}} .
$$

From (10) we now have

$$
\left|q_{n} \sum_{l \geq k+1} \varepsilon_{l}\left(q_{n_{l}} \alpha-p_{n_{l}}\right)\right|<2^{-k}
$$

Using (9) and (11) we obtain $\left|q_{n} \beta-S_{k}\right|<3 / 2^{k}$. Since $S_{k} \in \mathbb{Z}$ we have $\left\|q_{n} \beta\right\|<3 / 2^{k}$ for all $n>n_{3}$.

Remark 4. Let $\alpha$ be the real number given by $\left[0 ; 2,4, \ldots, 2^{n}, \ldots\right]$. Put $n_{l}:=l$, for $l \geq 0$, then $a_{n_{l}+1}=a_{l+1}=2^{l+1}$. Let $\left(\varepsilon_{l}\right)_{l \geq 1}$ be an arbitrary sequence of 0 's and 1's, and let $\beta$ be defined as in the above proof. Then we have $\beta \in E(\alpha)$ but $\beta$ is not an element of $\alpha \mathbb{Z}+\mathbb{Z}$ if $\varepsilon_{l}=1$ infinitely often. Moreover, by the choice of $\alpha$, we have $\limsup _{n \rightarrow \infty}\left(\left\|q_{n} \beta\right\|-\frac{1}{2} \theta_{n}\right)=0$ (see Remark 3).

Now as a direct consequence of Theorem 3 we have the following new characterization:

Proposition. Let $\alpha$ be an irrational number and let the group $E(\alpha)$ be defined as in Theorem 3. Then $\alpha$ has bounded partial quotients if and only if $E(\alpha)=$ $\alpha \mathbb{Z}+\mathbb{Z}$.

\section{REFERENCES}

[E] H. G. Eggleston, Sets of fractional dimensions which occur in some problems of number theory, Proc. London Math. Soc. (2) 54 (1952), 42-93.

[K] J. F. Koksma, Diophantische Approximationen, Springer, Berlin, 1936.

[L] G. Larcher, A convergence problem connected with continued fractions, Proc. Amer. Math. Soc. 103 (1988), 718-722.

[P] O. Perron, Die Lehre von den Kettenbrüchen, 2nd ed., Chelsea Publishing Company, New York, 1950.

[S] M. Stewart, Irregularities of uniform distribution, Acta Math. Acad. Sci. Hungar. 37 (1981), $185-221$.

Faculteit Wiskunde en Informatica, Universiteit van Amsterdam, Plantage MuiderGRACHT 24, TV AMSTERDAM, The NetherLANDS

Universite de Provence, Unite de Recherche Associé CNRS n ${ }^{\circ}$ 225, 3, Place Victor Hugo, 13331 Marseille Cedex 3, France 